\title{
General Medicine and Surgery for Dental Practitioners: Infections and Infection Control
}

\section{In Brief}

- All dental practitioners need to have a sound knowledge of the basics of infection and infection control.

- Common methods used to control cross infection are discussed.

- Safe working with sharps is considered.

- Some of the theoretical aspects of specific infections are highlighted.

\subsection{Introduction}

Healthcare-associated infections (HCAIs) are common in hospitals and clinics. In the UK in 2011, approximately $6.4 \%$ of hospital patients acquired infections as a result of procedures, devices or interventions [1]. The most common HCAIs involve the respiratory system, the urinary tract or at the site of surgery. In a dental setting, blood-borne viruses (BBVs) including hepatitis B virus (HBV), hepatitis $\mathrm{C}$ virus (HCV) and HIV, along with respiratory pathogens, present the highest risks of cross infection. There is a much lower risk of transmitting prions, but these agents are extremely difficult to inactivate and so pose unique problems in dentistry. New infectious agents frequently emerge, and dental practitioners must be prepared to respond to outbreaks of infection. 


\subsection{Points in the History}

In the medical history, it is sometimes possible to identify patients at high risk of transmitting or acquiring infection. However, many pathogens can exist in asymptomatic carriage states or are infectious before clinical disease symptoms appear. Therefore, the underlying principle of cross-infection control involves taking standard precautions to minimise the risk of transmission regardless of the health status of the patient or healthcare worker. Effective cross-infection control requires constant vigilance and involves the combined efforts of the entire dental team.

\subsection{Measures to Control Cross Infection}

Standard infection control precautions have been outlined by the NHS [2] and must be employed alongside local NHS policies and guidelines. These precautions are listed in Table 19.1 and described below. In addition, it is important to establish a culture of safety within a dental practice. This involves all practice staff and ensures that systems are in place for assessing and minimising the risk of cross infection, as well as for reporting incidents and potential exposure to infection. The design of the surgery must be considered carefully to ensure that dirty equipment is well separated from clean equipment and that the whole area is well ventilated and accessible for cleaning. Cross infection control depends on putting in place effective barriers to block the transmission of infectious agents. The major routes of transmission in the dental clinic are listed in Table 19.2.

\subsubsection{Hand Hygiene}

Good hand hygiene is one of the most important mechanisms to limit the spread of HCAIs. Every dental practice should have a hand hygiene policy. Sinks should be kept uncluttered and clean. Hands must be cleaned using a thorough procedure such as the Ayliffe technique [3], with scrubbing for between 20 and $30 \mathrm{~s}$ for a general wash or for to 2-3 min for a surgical scrub. Hands should be dried using clean disposable paper towels. Patients should be encouraged to clean their hands with an alcohol gel upon entering and leaving the treatment area.

Table 19.1 Standard cross infection precautions in a dental surgery
- Hand hygiene

- PPE (see text)

- Safe working with sharps

- Sterilisation and disinfection of dental instruments

- Surgery design and disinfection

- Dental unit waterlines

- Waste management

- Immunisations and screening 
Table 19.2 Possible routes of infective agent transmission
- Inoculation, e.g. sharps injuries or surgical procedures

- Inhalation of aerosols or droplets

- Absorption through intact or broken skin

- Absorption through mucous membranes including the eyes

- Ingestion

It is important to be aware of the "five moments for hand hygiene at the point of care". These "moments" are defined as:

- Before patient contact

- Before an aseptic task

- After body fluid exposure risk

- After patient contact

- After contact with patient surroundings

Effective handwashing is essential in the prevention of spread of methicillin resistant Staphylococcus aureus (MRSA). The alcohol gels are also effective against this bacterium. Another bacterium that has received significant attention recently is Clostridium difficile. This bacterium lives in the bowel of less than $5 \%$ of the adult population. Patients develop problems if they are brought into contact with contaminated surfaces or unwashed hands. Unlike MRSA, alcohol gels are not effective against $C$. difficile spores, and handwashing is therefore mandatory.

It is essential that healthcare workers remove all hand jewellery (although wedding bands are not included). Staff should be "bare below the elbows" and not wear a wrist watch. Nails should be kept short to make them easier to clean. Cuts and abrasions should be covered with waterproof adhesive dressings after handwashing.

The regular use of an emollient hand cream is important to prevent drying of the skin after frequent handwashing. Contact dermatitis can cause significant problems in susceptible individuals.

\subsubsection{Personal Protective Equipment}

The appropriate personal protective equipment (PPE) should be selected on the basis of a risk assessment and will depend on the procedure being carried out. Standard infection control precautions call for the use of gloves, aprons or gowns and mouth/eye protection such as masks or goggles for any procedures that involve direct contact with patients' body fluids. Note that PPE will only reduce the risk of transmission and cannot provide a failsafe barrier. All PPE falls under medical device regulations and must be approved and marked with the European CE mark (Fig. 19.1). 


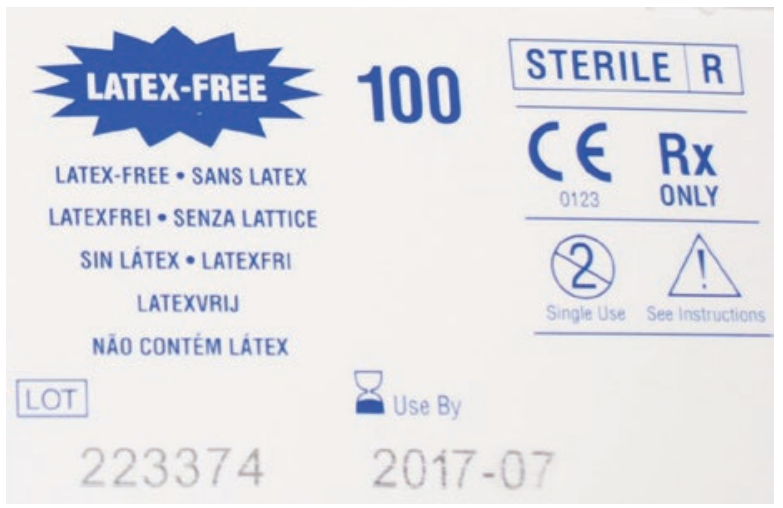

Fig. 19.1 Markings on a packet of endodontic syringes and needles, including the CE mark and an indication that the product is latex-free

Gloves should be worn for all dental procedures, including mopping up spills or handling waste. Hands should be washed before and after wearing gloves. Singleuse gloves must be changed between patients and should never be washed as they will lose their protective function. They should not be worn outside the clinical area. Most organisations now routinely use latex-free gloves. Allergies to natural rubber latex gloves occur in 6-18\% of healthcare workers and cause symptoms such as dermatitis, asthma and occasionally anaphylaxis. Patients with known allergies to latex gloves should be identified in the medical history, and their notes should be clearly labelled. Latex gloves should be avoided for these patients or for healthcare workers with latex allergies. Alternatives to latex include nitrile, polychloroprene (neoprene), vinyl or copolymer gloves. Look for "latex-free" markings on gloves or other items in the surgery (Fig. 19.1).

Masks are recommended for all dental procedures to protect against droplets and aerosols and should be tight-fitting around the mouth and nose. Masks are single use and should be discarded between patients. During care of patients with respiratory infections, particulate respirator masks should be worn since standard surgical masks do not provide adequate protection against small particles. British standard EN149:2001 (amended in 2009) classifies disposable filtering facepiece (FFP) respirators into three categories (FFP1-3), of which FFP3 provides the highest level of protection. FFP2 respirators should be worn when treating patients with active tuberculosis. During pandemic influenza, any aerosol-generating procedures on infected patients should be performed only with an FFP3 respirator, and the user must have undertaken a respirator fit test prior to using it. Elective procedures should be postponed until the infection has resolved.

Eyes must be protected by face shields or goggles during all dental procedures. If goggles are selected, they should have side protection conforming to BS EN166:1988 and should be cleaned according to manufacturers' instructions. Visors are usually single use and disposable. Reusable face shields must be cleaned with disinfectant. 
Tunics and uniforms should be washed daily on a hot cycle of $60{ }^{\circ} \mathrm{C}$. Tunics and uniforms are not considered PPE since they are made of absorbent materials and provide little protection against pathogens. Single-use plastic aprons should be worn to protect against spatter and must be changed between patients. For minor oral surgery, surgical gowns with tight-fitting cuffs should be worn to protect both the clinician and the patient from transmission of microorganisms on the skin.

\subsubsection{Safe Working With Sharps}

"Sharps" injuries are amongst the most common type of injury in the dental surgery. It is estimated that approximately half of these are preventable. Puncturing the skin is the major route of transmission for BBVs. It is essential, therefore, that appropriate risk assessments, accident reporting procedures and, most importantly, safe working practices are applied for work that involves sharps.

Many sharps injuries occur outside the mouth during re-sheathing, dismantling or disposal of needles or during cleaning of sharp instruments such as burs and probe tips. When working inside the mouth, the use of a mirror or other device rather than fingers to retract the tongue and cheeks reduces risk. Needles should not be re-sheathed, recapped, bent or disassembled after use. Consider the use of a "sharp-safe" needle design that does not require re-sheathing (Fig. 19.2). Passing instruments from hand to hand during dental procedures should be avoided. Instruments are best transferred in a receiver. Used sharps must be placed in a container conforming to UN3291 and BS7320 standards. These containers need to be placed out of reach of children and should not be filled above the indicated level. Sharps must never be removed from disposal containers.

The European Council Directive 2010/32/EU [4] which was published in June 2010 and implemented in May 2013 dictates that "the practice of recapping shall be banned with immediate effect" and that staff require training in the "correct use of medical devices incorporating sharps protection mechanisms".

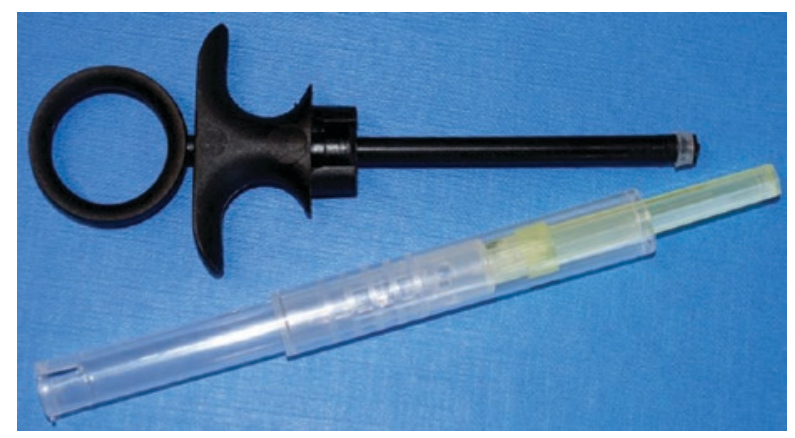

Fig. 19.2 Example of a "sharp-safe" syringe design. The outer plastic is drawn down to cover the needle after use. If the outer sleeve is clicked into place once, the sleeve can be retracted again for further use of the syringe. A double click permanently locks the cover into position 


\subsubsection{Sterilisation and Disinfection of Dental Instruments}

Sterilisation is aimed at the complete removal of viable organisms, including bacterial spores. The simplest method of sterilisation involves the use of steam under pressure, for example, at a temperature of $121{ }^{\circ} \mathrm{C}$ for $15-30 \mathrm{~min}$ or at $134{ }^{\circ} \mathrm{C}$ for 3-4 min in an autoclave. It is necessary to clean all devices thoroughly prior to autoclaving since particles of dirt can protect microorganisms from the autoclave cycle. To ensure that the steriliser is functioning correctly, automatic control tests must be performed daily, and records should be retained in a logbook for at least 2 years. Chemical process indicators, such as autoclave tape, sterilisation packaging or bags containing an indicator are useful to identify items that have been sterilised but cannot be used to validate the autoclave cycle. Steam sterilisation is not appropriate for all equipment, and chemical sterilisation with ethylene oxide gas, formaldehyde gas, hydrogen peroxide gas, liquid peracetic acid or ozone can be used as an alternative. Validated chemical sterilisation methods are not usually available in dental surgeries and are most easily obtained through a specialist contractor. Singleuse devices provide a simple alternative to sterilisation of sensitive equipment and are indicated by a clear logo, as shown in Fig. 19.3.

Disinfection processes aim for a reduction in microbial load to levels that are considered acceptable. Cleaning and disinfection may be performed manually or, preferably, using automated systems. Ultrasonic baths provide excellent cleaning for intricate, jointed or serrated stainless steel instruments. Thermal washer disinfectors include a high-temperature step (typically $90{ }^{\circ} \mathrm{C}$ for $1 \mathrm{~min}$ ), which significantly reduces the microbial contamination of devices. The final rinsing should be performed with high-quality water, such as reverse osmosis (RO)-treated water.

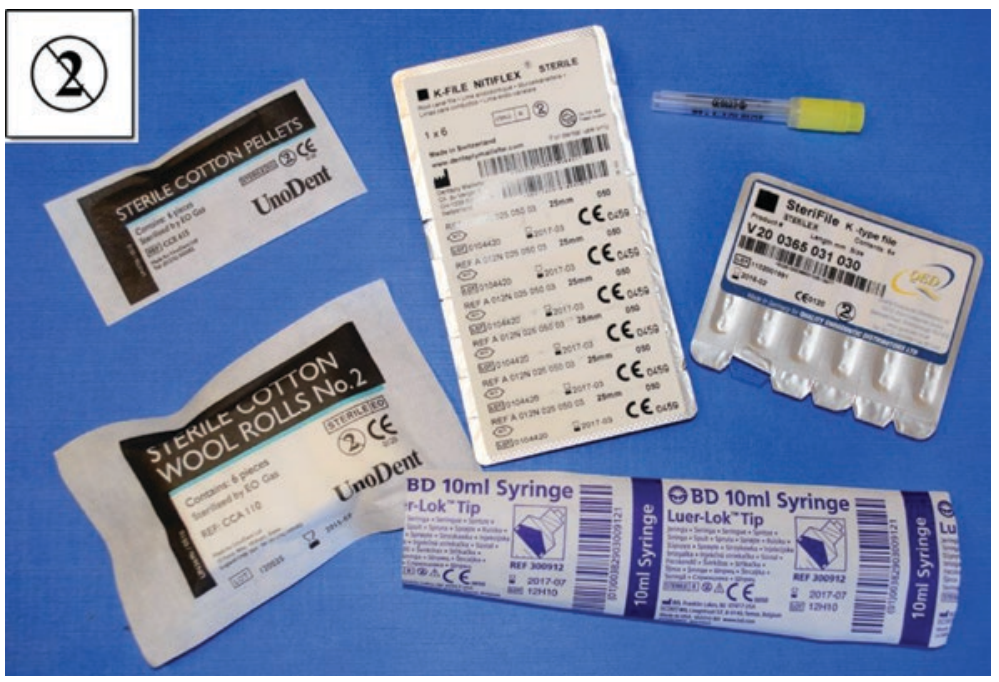

Fig. 19.3 Single-use items labelled with the appropriate logo 


\subsection{Surgery Design and Disinfection}

Dental surgeries should be designed with a view to easy cleaning and provide adequate workspace (17 $\mathrm{m}^{2}$ is usually considered sufficient), good ventilation, and separation of clean and dirty zones. The layout should provide access for disabled patients or staff and an easy escape route in case of emergency.

In ideal surgery design, the room should be well ventilated by open windows or air conditioning, with a fresh air supply of at least 5-8 L/s per occupant. Air should be ventilated outside the building and filters need to be replaced regularly. The floor should be non-slip and should curve up the wall by $7.5 \mathrm{~cm}$ to allow easy cleaning. It is important to clean and disinfect the floor daily. Dental chairs and other furnishings within the surgery should be upholstered with wipeable material and should be free from visible damage. A sink must be available within the room and should be kept clean and clear of clutter. Elbow-operated or motion-sensitive taps are preferred. The water jet must not be directed straight down the plughole as this creates aerosols. Taps that are only used sporadically should be flushed weekly to limit the accumulation of microorganisms in stagnant water.

All areas of a dental practice should be cleaned regularly. The patient treatment area should be cleaned between each patient using disposable alcohol hand wipes or clean microfibre materials. The treatment area includes local work surfaces, dental chairs, inspection lights, hand controls, trolleys, spittoons, aspirators, sinks and taps. Computer keyboards in clinical areas should be of the "easy-clean" type or should be fitted with wipeable covers. For blood spillages, $\mathrm{NaOCl}$ (sodium hypochlorite) at a concentration of $1 \%$, yielding at least 1000 ppm free chlorine, must be applied for at least $5 \mathrm{~min}$. Sodium hypochlorite is not recommended for use on metal surfaces due to the risk of corrosion.

"Clean" and "dirty zones" must be kept well-separated, and a logical workflow from dirty to clean should be established. Ideally, decontamination areas should be in a separate room from patient treatment areas. Where this is not possible, controls are needed to prevent cross-contamination between the patient and the dirty equipment. Manual cleaning and decontamination procedures liable to generate aerosols should not be performed whilst the patient is present. The physical layout of the surgery should facilitate the easy movement of devices from dirty to clean areas. For example, an area of bench just inside the dirty zone should be designated as the receiving area. Washing and rinsing sinks and/or equipment should be sited close to the receiving area. A steriliser should be located away from the washing area to avoid contamination of freshly sterilised instruments. Once sterilised, instruments should be stored in a clean area well away from the dirty zone and preferably in a different room.

\subsubsection{Dental Unit Waterlines (DUWLs)}

If not maintained adequately, DUWLs can harbour microbial pathogens such as pseudomonads or Legionella. These organisms grow as biofilms on the internal surfaces of water bottles and tubing, where they are protected against chemical cleaning 
agents. To prevent the accumulation of biofilms, systems should be drained down at the end of each day. If present, self-contained water bottles should be removed and emptied. DUWLs should be fed with RO-treated water and should be flushed regularly. Flushing for $2 \mathrm{~min}$ at the start and end of each day, and for 20-30 s between patients, is recommended. Disinfection of DUWLs should be performed periodically and additionally if visible contamination is observed. A number of different agents are available for disinfection of DUWLs, and the appropriate agent should be selected on the basis of the manufacturer's instructions. All hand pieces, ultrasonic scalers and waterlines should be fitted with anti-retraction valves, and these should be maintained and checked periodically. In-line filters used in DUWLs should be checked at intervals or, if they are the disposable type, should be changed daily.

\subsubsection{Waste Management}

Any waste containing human or animal tissue, blood or bodily fluids, drugs, swabs or dressings, or other potentially infectious material, must be clearly labelled as "clinical waste" and segregated from non-clinical waste. Used disposable syringes, needles or other sharp instruments must be deposited in a "sharp's box". Teeth containing amalgam should not be incinerated as the amalgam will release toxic mercury compounds. Extracted teeth with amalgam fillings must be stored in a rigid container and removed by a specialist contractor. Amalgam removed from the mouth must be collected using an amalgam separator and collected in the waste line of the apparatus.

Waste should be stored in a dedicated area, away from public access, prior to collection. Excessive waste build up should be avoided. A consignment note is required for all collections of hazardous waste, and the appropriate sections must be completed by the waste producer and the waste carrier. Consignment notes should be retained by the practice for at least 3 years. All practices that produce over $500 \mathrm{~kg}$ of waste per annum must register with the Environment Agency as a hazardous waste producer.

\subsection{Immunisations and Screening}

Immunisations provide an additional layer of protection against the transmission of certain pathogens from patients to dental care professionals. In a dental practice, it is recommended that one member of staff is designated to maintain confidential records of immunisations and healthcare screening of all workers in the practice. In the UK, all healthcare workers should stay up to date with routine immunisations against diphtheria, tetanus, polio, measles, mumps and rubella. Annual vaccination against seasonal influenza is also strongly recommended. Clinical staff who have direct contact with body fluids of patients should additionally be vaccinated against tuberculosis, varicella zoster virus (if not immune already) and HBV. Recommended immunisations are summarised in Table 19.3. 
Table 19.3 Recommended immunisations

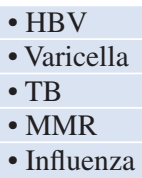

Healthcare workers are significantly more likely than the general public to be exposed to tuberculosis (TB). All healthcare workers entering the NHS, including students on clinical dentistry courses, are required to complete a TB screen or health check before treating patients [5]. Varicella causes chickenpox or shingles and is highly infectious. If a woman is infected in the first 20 weeks of pregnancy, damage to the foetus can result. It is therefore recommended that female healthcare workers of childbearing age should receive the vaccine if they are not already immune.

Vaccination against HBV is essential for healthcare workers due to the high risk of exposure to infection, and workers must obtain a certificate of immunity prior to performing exposure prone procedures. A recombinant vaccine is administered in three doses, followed by a blood test for antibodies to hepatitis B surface antigen. Approximately $10-15 \%$ of adults respond poorly to the vaccine, and an additional dose may be necessary. In all cases, a booster is required 5 years after the initial course of vaccination. Students entering UK dental schools are required to be screened for HCV and HIV infection [5]. Students who are carriers of HBV or HCV and are subsequently treated will become eligible to undertake exposure-prone procedures provided that the disease is appropriately monitored.

\subsection{Infectious Agents of Concern to the Dental Practitioner}

The major infectious agents of concern in a dental clinic are listed in Table 19.4 and described below.

\subsubsection{Blood-Borne Viruses}

In the 1970s and 1980s, before widespread immunisation, studies in the USA revealed that healthcare workers had a significantly higher rate of HBV infection than the general population [6]. The emergence of HIV in the 1980s prompted the US Centers for Disease Control to issue guidance about universal precautions for avoiding direct contact with blood or blood-contaminated secretions from all patients independent of infection status [7]. These measures have subsequently been modified and merged with other guidance to create a set of standard precautions to be used with all patients at all times, which have been outlined above. Since the introduction of such measures in both the USA and the UK, rates of BBV infection amongst healthcare workers have decreased to the point where they are no higher than the general population [8]. Nevertheless, there continues to be occasional reports of transmission of BBVs between patients and dental professionals. 
Table 19.4 Infectious agents of concern in dentistry

- BBVs

- Respiratory viruses

- TB

- MRSA

- Pseudomonas

- Legionella

- Prions

Hepatitis B virus is endemic in the UK but at a low prevalence rate of $0.1-0.5 \%$. The incidence of chronic $\mathrm{HBV}$ is increasing in the UK as a result of immigration from parts of the world where HBV prevalence is much higher. Infection with HBV involves an acute phase, lasting around 75 days, in which symptoms including fever, nausea, abdominal pain, dark urine, grey-coloured faeces, joint pain and jaundice often appear. The likelihood that acute infection will progress to chronic or carriage state varies from around $90 \%$ of neonates, $25 \%$ of children or $5 \%$ of adults that acquire HBV. Fortunately, there is an effective vaccine against HBV, which gives approximately $95 \%$ protection, and all healthcare professionals should receive this vaccine.

Approximately 216,000 individuals in the UK are chronically infected with hepatitis $\mathrm{C}$ virus (HCV). Intravenous drug users are at particularly high risk of acquiring infections: in some parts of the UK, HCV infection rates in this group exceed $50 \%$. Following infection, around $80 \%$ of individuals remain symptom-free, whilst others exhibit symptoms similar to those of HBV. The incubation period for $\mathrm{HCV}$ is 2 weeks to 6 months. Chronic disease occurs in 75-85\% of individuals infected with $\mathrm{HCV}$, and of these around 60-70\% develop chronic liver disease. A further 5-20\% of individuals develop cirrhosis, and a small proportion (1-5\%) die from cirrhosis or liver cancer. There is no vaccine for HCV, but the disease is curable with a combination of antiviral agents.

At the end of 2011, around 96,000 people in the UK were infected with HIV. Of these, almost one quarter were unaware of their infection. Following infection, most individuals experience a short "flu-like" illness, followed by a period of several years without symptoms. Antiretroviral therapy (ART) is extremely effective for delaying the onset of late-stage HIV infection, or AIDS. The majority of individuals receiving antiretroviral therapy are virally suppressed and hence not infectious. The incidence of TB in HIV patients has declined from around 30\% in 2002 to $8.8 \%$ in 2010 but nevertheless remains higher than the general population.

\subsubsection{Respiratory Viruses}

Several different viruses cause respiratory infections, including the common cold (rhinovirus), coronaviruses, respiratory syncytial virus, adenoviruses and influenza. Influenza $\mathrm{A}$ is a particular concern since infection can be severe. In some cases, influenza A leads to secondary complications such as bronchitis and secondary 
pneumonia, which can be life-threatening. Seasonal variants of influenza appear each year, and annual vaccination is recommended. Occasionally, larger variations in the structure of the virus lead to rapidly spreading strains that cause global episodes of infection or pandemics.

Following the pandemic "flu of 2009", the UK Department of Health developed a UK Influenza Pandemic Preparedness Strategy to put in place structures for rapid responses to a new pandemic. During a pandemic, dental professionals should contact patients $24 \mathrm{~h}$ in advance of appointments to ensure they are free of symptoms. Treatment of patients with infection should be delayed until symptoms abate if at all possible. Where emergency dental care is required for infected patients, care should be provided by practices selected and supported by primary care trusts or the appointed Commissioner of services. Infected patients must be separated from noninfected individuals in segregated waiting areas. Equipment that generates aerosols should be avoided if possible in this situation. Treatment should not be administered by healthcare workers who are at high risk of complications from influenza infection, including pregnant women and the immunocompromised.

\subsubsection{Mycobacterium tuberculosis}

Around one third of the world's population is infected with Mycobacterium tuberculosis, the primary causative agent of TB. In the UK, around 9000 cases of TB are reported each year, mostly in major cities. Symptoms may not occur until months or even years after infection. Symptoms of pulmonary TB include severe coughing for 3 weeks or more productive of sputum and sometimes blood, breathlessness which gradually worsens, lack of appetite and weight loss, temperatures in excess of $38{ }^{\circ} \mathrm{C}$, night sweats, extreme tiredness or pain lasting more than 3 weeks. Extrapulmonary TB occurs most commonly in HIV patients and can affect the lymph nodes, the bones and joints, the gastrointestinal tract, the genitourinary tract or the central nervous system. Treatment with a prolonged course of antibiotics is usually successful, although the number of cases of multidrug-resistant M. tuberculosis has gradually risen.

\subsubsection{Methicillin-Resistant Staphylococcus aureus (MRSA)}

Staphylococcus aureus is a commensal of the skin or nasopharynx and is detected in the oral cavities of around 30\% of people [9]. Acquired resistance to antibiotics was first noted in S. aureus in the 1940s, shortly after the introduction of penicillin, and the spectrum of antibiotic resistance has been increasing ever since. Strains of MRSA are resistant to multiple antibiotics, and some $S$. aureus infections are now very difficult to treat. For many years, hospital-acquired MRSA (HA-MRSA) has been the major threat in the UK. However, more recently community-acquired MRSA (CA-MRSA) has emerged as a significant problem in the USA, and it is possible that this will also take hold in the UK. Whereas HA-MRSA tends to affect 
elderly or immunocompromised patients in healthcare settings, CA-MRSA often occurs in young, otherwise healthy individuals. S. aureus is primarily transmitted through contact and causes a wide range of different infections ranging from superficial lesions to deep-seated infections such as septic arthritis, endocarditis or pneumonia. Toxins produced by $S$. aureus can cause food poisoning or toxic shock syndrome. In the mouth, $S$. aureus may be responsible for angular cheilitis, parotitis, osteomyelitis or mucositis. Standard cross infection control measures should restrict the spread of MRSA, and hand hygiene is particularly important.

\subsubsection{Pseudomonads}

Pseudomonas and related genera are very common in water systems including dental unit waterlines. These gram-negative bacteria are opportunistic pathogens, responsible for lung infections or for infections of wounds or burns. Pseudomonads are of significant concern in augmented care units, such as burns or intensive care units. There is little evidence, however, that these organisms have been transmitted during dental treatment.

\subsubsection{Legionella}

Legionnaire's disease was first identified following a convention of the American Legion in Philadelphia in 1976, where 182 attendees fell ill with symptoms of pneumonia and 28 of those died. Legionella pneumophila lives in water systems and can survive within amoebae, where it is protected from chlorination. Inadequate maintenance of water systems can lead to outbreaks of disease. Dental unit waterlines may harbour Legionella and must be cleaned regularly. All dental practices in the UK are now required to have a risk assessment for Legionella [10]. It is recommended that practice managers engage with organisations that are registered members of the Legionella Control Association to ensure that working practices are safe and comply with legal requirements.

\subsubsection{Prions}

Prions are infectious proteins that can nucleate the misfolding of natural proteins in brain tissue. This process leads to progressive neurodegeneration and ultimately to death. An epidemic of prion disease in cattle, bovine spongiform encephalopathy (BSE, or mad cow disease), occurred in the UK between 1985 and 1996 and resulted in the slaughter of 4.4 million cows. Subsequently, a new variant of CreutzfeldtJakob disease (vCJD) was identified in humans and has caused 176 deaths up to the end of 2012. Prion diseases are extremely rare, but the infectious agent is highly resistant to standard disinfection and sterilisation protocols, even including steam sterilisation. Harsh chemical treatments such as 1-2M sodium hydroxide for $1 \mathrm{~h}$ at 
room temperature or immersion in greater than 20,000 ppm sodium hypochlorite 1 for $1 \mathrm{~h}$ at room temperature may inactivate prions, as will extended steam sterilisation, for example, $121{ }^{\circ} \mathrm{C}$ for $1 \mathrm{~h}$, or $134{ }^{\circ} \mathrm{C}$ for $18 \mathrm{~min}$. However, these procedures may not be compatible with manufacturers' recommendations for devices and are likely to be most appropriate for known or suspected cases of prion disease. Alternatively, single-use devices are a good option if there is a significant risk of prion transmission.

\subsection{Summary}

Good management systems are essential for maintaining effective cross infection control in a dental practice. Responsibilities for overseeing cleaning, sterilisation and maintenance of equipment should be allocated to individual members of staff. However, all staff in the surgery need to be aware of the importance of cross infection control and the underlying principles behind maintaining a safe working environment. Understanding the modes of transmission of infectious agents is important in order to put in place appropriate barriers against transmission. Regular reviews of procedures are required to ensure that systems are functioning properly and that they conform to the latest local and national guidelines and legislation.

\section{References}

1. Health Protection Agency. English national point prevalence survey on healthcare-associated infections and antimicrobial use, 2011: preliminary data. 2012.

2. NHS, NHS professionals-standard infection control precautions. 2010. www.nhsprofessionals.nhs.uk/download/comms/CG1_NHSP_Standard_Infection_Control_Precautions_v3.pdf.

3. Pankhurst CL, Coulter WA. Basic guide to infection prevention and control in dentistry. In: Chapter 5 Hand Hygiene. Hoboken: Wiley. p. 64-75.

4. European Council Directive 2010/32/EU implementing the framework agreement on prevention from sharp injuries in the hospital and healthcare sector concluded by HOSPEEM and EPSU. http://eur-lex.europa.eu/LexUriServ/LexUriServ.do?uri=OJ:L:2010:134:0066:0072:E $\mathrm{N}:$ PDF.

5. Department of Health, Health Clearance for tuberculosis, hepatitis B, hepatitis C and HIV: New healthcare workers. 2007.

6. Cleveland JL, et al. Factors associated with hepatitis B vaccine response among dentists. J Dent Res. 1994;73(5):1029-35.

7. Siegel JD, et al. Guideline for isolation precautions: preventing transmission of infectious agents in healthcare settings. 2007. http://www.cdc.gov/ncidod/dhgp/pdf/ioslation2007.pdf.

8. Gunson RN, et al. Hepatitis B virus (HBV) and hepatitis C virus (HCV) infections in health care workers (HCWs): guidelines for prevention of transmission of $\mathrm{HBV}$ and $\mathrm{HCV}$ from $\mathrm{HCW}$ to patients. J Clin Virol. 2003;27(3):213-30.

9. O'Leary E, Lockhart DE, Smith A. Coming to a practice near you? Community-acquired meticillin-resistant Staphylococcus aureus (CA-MRSA). Dent Update. 2011;38(4):254-6. 259-60

10. Department of Health, Health technical memorandum 01-05-decontamination in primary care dental practices. 2009. 